PROCEEDINGS OF THE

AMERICAN MATHEMATICAL SOCIETY

Volume 127, Number 2, February 1999, Pages 401-407

S 0002-9939(99)05120-5

\title{
PERIODIC SOLUTIONS OF DAMPED DIFFERENTIAL SYSTEMS WITH REPULSIVE SINGULAR FORCES
}

\author{
MEIRONG ZHANG
}

(Communicated by Hal L. Smith)

\begin{abstract}
We consider the periodic boundary value problem for the singular differential system: $u^{\prime \prime}+(\nabla F(u))^{\prime}+\nabla G(u)=h(t)$, where $F \in C^{2}\left(\mathbb{R}^{N}, \mathbb{R}\right)$, $G \in C^{1}\left(\mathbb{R}^{N} \backslash\{0\}, \mathbb{R}\right)$, and $h \in L^{1}\left([0, T], \mathbb{R}^{N}\right)$. The singular potential $G(u)$ is of repulsive type in the sense that $G(u) \rightarrow+\infty$ as $u \rightarrow 0$. Under HabetsSanchez's strong force condition on $G(u)$ at the origin, the existence results, obtained by coincidence degree in this paper, have no restriction on the damping forces $(\nabla F(u))^{\prime}$. Meanwhile, some quadratic growth of the restoring potentials $G(u)$ at infinity is allowed.
\end{abstract}

\section{INTRODUCTION}

In this paper, we study existence results to the periodic boundary value problem for the repulsive singular differential system:

$$
\begin{aligned}
& u^{\prime \prime}+(\nabla F(u))^{\prime}+\nabla G(u)=h(t), \\
& u(0)-u(T)=u^{\prime}(0)-u^{\prime}(T)=0,
\end{aligned}
$$

where $F \in C^{2}\left(\mathbb{R}^{N}, \mathbb{R}\right), G \in C^{1}\left(\mathbb{R}^{N} \backslash\{0\}, \mathbb{R}\right)$, and $h \in L^{1}\left([0, T], \mathbb{R}^{N}\right)$. The potential $G(u)$ is singular (at 0 ) in the sense that $G(u)$ becomes infinity when $u$ tends to 0 . From the physical meaning of Eq. (1.1), the singular potential $G(u)$ is said to be of attractive type (repulsive type, respectively) when $G(u) \rightarrow-\infty(G(u) \rightarrow+\infty$, respectively) as $u \rightarrow 0$; see Ambrosetti [1].

By a solution $u(\cdot)$ of $(1.1)-(1.2)$, we mean that $u \in W^{2,1}\left([0, T], \mathbb{R}^{N}\right)$ such that $u(t) \neq 0$ for all $t \in[0, T]$ and satisfies the boundary condition (2.2) and Eq. (2.1) for a.e. $t \in[0, T]$.

When no damping is presented in Eq. (1.1), i.e., $F(u) \equiv 0$, the problem (1.1)(1.2) is of variational structure and can be handled by critical point theory. Such an approach is initiated by Gordon [3], who introduced a strong force condition on $G(u)$ at 0 to get some compactness for the corresponding action functionals. We

Received by the editors September 23, 1996.

1991 Mathematics Subject Classification. Primary 34C15, 34C25.

Key words and phrases. Singular force, strong force condition, damped system, coincidence degree.

The author is supported by the National Natural Science Foundation of China and the Tsinghua University Education Foundation.

(C)1999 American Mathematical Society 
refer to Ambrosetti [2] for the attractive case and to Coti Zelati [2] and Solimini [10] for the repulsive case.

When damping is presented in Eq. (1.1), another approach is also developed by using the Leray-Schauder degree, or its variant, the coincidence degree (see Mawhin [8], [9]). For the one-dimensional systems, we refer to Laser and Solimini [6] and Habets and Sanchez [4]. Of particular interest, when $F(u) \equiv \frac{1}{2} c|u|^{2}$ and some necessary assumptions on $G(u)$ at 0 are assumed, Mawhin's result in [9] allows the repulsive potentials $G(u)$ to have some quadratic growth at infinity. The present author has recently generalized this result, in [11], to general dampings $F(u)$ and potentials $G(t, u)$, periodic in $t$. An interesting application to the Brillouin electron beam focusing system is also given in [11]. For the higher dimensional systems, Habets and Sanchez [5] introduced a different strong force condition on $G(u)$ at the origin from the viewpoint of degree theory. However, the existence results in [5] mainly depend upon a strict damping force condition, i.e., there exists some constant $a>0$ such that either

or

$$
\frac{\partial^{2} F}{\partial u^{2}}(u) \geq a I_{N} \quad \text { for all } u \in \mathbb{R}^{N}
$$

$$
\frac{\partial^{2} F}{\partial u^{2}}(u) \leq-a I_{N} \quad \text { for all } u \in \mathbb{R}^{N} .
$$

In this paper we consider higher dimensional systems. Under Habets-Sanchez's strong force condition on $G(u)$ at 0 , the existence results in this paper can be applied to any damping forces without imposing more conditions on singular potentials $G(u)$ than those in Habets and Sanchez [5]. Meanwhile, some quadratic growth of the restoring potentials $G(u)$ at infinity is also allowed.

\section{MAin Result}

In the sequel, we use $\cdot$ and $|\cdot|$ to denote the Euclidean inner product and norm, respectively, in $\mathbb{R}^{N}$. Meanwhile, the $L^{p}$-norm in the space $L^{p}\left([0, T], \mathbb{R}^{N}\right)$, $1 \leq p \leq \infty$, is denoted by $\|\cdot\|_{p}$. For $h \in L^{1}\left([0, T], \mathbb{R}^{N}\right)$, the mean value is defined by

$$
\bar{h}=\frac{1}{T} \int_{0}^{T} h(t) d t .
$$

As mentioned in Section 1, we consider the following boundary value problem:

$$
\begin{gathered}
u^{\prime \prime}+(\nabla F(u))^{\prime}+\nabla G(u)=h(t), \quad \text { a.e. } t \in[0, T], \\
u(0)-u(T)=u^{\prime}(0)-u^{\prime}(T)=0,
\end{gathered}
$$

where $F \in C^{2}\left(\mathbb{R}^{N}, \mathbb{R}\right), G \in C^{1}\left(\mathbb{R}^{N} \backslash\{0\}, \mathbb{R}\right)$, and $h \in L^{1}\left([0, T], \mathbb{R}^{N}\right)$.

We impose the conditions on $G$ as follows:

(G1) $\lim _{u \rightarrow 0} u \cdot \nabla G(u)=-\infty$.

(G2) Habets-Sanchez's Strong Force Condition at 0 [5]: There exists $\varphi \in$ $C^{1}\left(\mathbb{R}^{N} \backslash\{0\}, \mathbb{R}\right)$ such that: (i) $\lim _{u \rightarrow 0} \varphi(u)=+\infty$; and (ii) $|\nabla \varphi(u)|^{2} \leq|u \cdot \nabla G(u)|+c_{1}$ when $0<|u| \ll 1$. Here and henceforth $c_{i}$ represent positive constants.

(G3) There exist constants $c_{2}, c_{3}$ such that

$$
u \cdot \nabla G(u) \leq c_{2}|u|^{2}+c_{3}, \quad \forall u \in \mathbb{R}^{N} \backslash\{0\},
$$


and $c_{2}$ satisfies

$$
c_{2}<\pi^{2} / T^{2}
$$

Theorem 1. Besides the conditions (G1), (G2) and (G3), we assume further that the following (G4) and (G5) are satisfied:

(G4) There exists a constant $R_{1}>0$ such that, for any solution $u(\cdot)$ of the problem

$$
\begin{gathered}
u^{\prime \prime}+\lambda\left((\nabla F(u))^{\prime}+\nabla G(u)\right)=\lambda h(t), \quad \lambda \in(0,1], \\
u(0)-u(T)=u^{\prime}(0)-u^{\prime}(T)=0,
\end{gathered}
$$

one has $|u(\tau)|<R_{1} \quad$ for some $\tau \in[0, T]$.

(G5) The topological degree

$$
\operatorname{deg}\left(\nabla G, \Omega_{r, R}, \bar{h}\right) \neq 0
$$

for all $0<r \ll 1$ and some sufficiently large $R \gg 1$, where $\Omega_{r, R}=\left\{x \in \mathbb{R}^{N}: r<\right.$ $|x|<R\}$.

Then (2.1)-(2.2) has at least one solution.

Proof. The conditions (G4) and (G5) are natural from the viewpoint of coincidence degree. (G4) and (G5) are (or implicitly are) assumed in previous works [4], [5]. For more details on our assumptions, see the next section.

The proof is based on the following result of Mawhin [8] from coincidence degree theory (see also Mawhin [9]):

Let $f=f(t, x, y):[0, T] \times \mathbb{R}^{N} \times \mathbb{R}^{N} \rightarrow \mathbb{R}^{N}$ be an $L^{1}$-Carathéodory function. Let $\Omega \subset \mathbb{R}^{N} \times \mathbb{R}^{N}$ be an open bounded set such that

(i) for any solution $x$ of

$$
(P)_{\lambda} \quad\left\{\begin{array}{l}
x^{\prime \prime}=\lambda f\left(t, x, x^{\prime}\right), \quad \lambda \in(0,1] \\
x(0)-x(T)=x^{\prime}(0)-x^{\prime}(T)=0
\end{array}\right.
$$

one has

$$
\left(x(t), x^{\prime}(t)\right) \in \bar{\Omega}, \forall t \in[0, T] \quad \Longrightarrow \quad\left(x(t), x^{\prime}(t)\right) \in \Omega, \forall t \in[0, T] .
$$

(ii) $\operatorname{deg}\left(\bar{f}, \Omega \cap\left(\mathbb{R}^{N} \times\{0\}\right), 0\right) \neq 0$, where

$$
\bar{f}(x)=\frac{1}{T} \int_{0}^{T} f(t, x, 0) d t .
$$

Then the problem $(P)_{1}$ has at least one solution $x(\cdot)$ such that $\left(x(t), x^{\prime}(t)\right) \in \Omega$ for all $t \in[0, T]$.

So the theorem is proved by obtaining a priori bounds for all solutions of (2.5)(2.6). Let $u(\cdot)$ be a solution of $(2.5)-(2.6)$ for some $\lambda \in(0,1]$. The estimates for the $C^{1}$-norm of $u(\cdot)$ are completed in several steps.

Step 1. Upper bounds for $\|u\|_{\infty}$ and $\left\|u^{\prime}\right\|_{2}$ follow from (G3) and (G4).

Multiplying (2.5) with $u$, we obtain

$$
-u \cdot u^{\prime \prime}=\lambda u \cdot(\nabla F(u))^{\prime}+\lambda u \cdot \nabla G(u)-\lambda h \cdot u .
$$

Note that the equalities

$$
-u \cdot u^{\prime \prime}=u^{\prime} \cdot u^{\prime}-\frac{d}{d t} u \cdot u^{\prime}, \quad \text { a.e. } t \in[0, T]
$$




$$
u \cdot(\nabla F(u))^{\prime}=\frac{d}{d t}(u \cdot \nabla F(u)-F(u))
$$

hold. We get, by integrating (2.7) over $[0, T]$ and using the boundary conditions (2.6),

$$
\begin{aligned}
\left\|u^{\prime}\right\|_{2}^{2} & =\lambda \int_{0}^{T} u \cdot \nabla G(u) d t-\lambda \int_{0}^{T} h \cdot u d t \\
& \leq \lambda \int_{0}^{T}\left(c_{2}|u|^{2}+c_{3}\right) d t+\lambda\|h\|_{1}\|u\|_{\infty} \\
& =\lambda\left(c_{2}\|u\|_{2}^{2}+\|h\|_{1}\|u\|_{\infty}+T c_{3}\right),
\end{aligned}
$$

where $(2.3)$ is used.

On the one hand, by (G4) and the mean value theorem, we have

$$
|u(t)|=\left|u(\tau)+\int_{\tau}^{t} u^{\prime}(s) d s\right| \leq R_{1}+\sqrt{T}\left\|u^{\prime}\right\|_{2}
$$

for all $t$. Thus

$$
\|u\|_{\infty} \leq R_{1}+\sqrt{T}\left\|u^{\prime}\right\|_{2} .
$$

On the other hand, we periodically extend the domain of $u(\cdot)$ to the whole $\mathbb{R}$ and define

$$
v(t)=u(t+\tau)-u(\tau),
$$

where $\tau$ is as in (G4). Then $v \in H^{1}(0, T)$ satisfies the Dirichlet boundary condition

$$
v(0)=v(T)=0 \text {. }
$$

Thus

which, in turn, implies

$$
\|v\|_{2}^{2} \leq \frac{T^{2}}{\pi^{2}}\left\|v^{\prime}\right\|_{2}^{2}=\frac{T^{2}}{\pi^{2}}\left\|u^{\prime}\right\|_{2}^{2}
$$

$$
\begin{aligned}
\|u\|_{2}^{2} & =\|u(\tau)+v\|_{2}^{2} \\
& \leq\left(\sqrt{T}|u(\tau)|+\|v\|_{2}\right)^{2} \\
& \leq T R_{1}^{2}+2 \sqrt{T} R_{1} \frac{T}{\pi}\left\|u^{\prime}\right\|_{2}+\frac{T^{2}}{\pi^{2}}\left\|u^{\prime}\right\|_{2}^{2} .
\end{aligned}
$$

Under the assumption (2.4), it follows from (2.8), (2.9) and (2.10) that there exist some positive constants $R_{2}, R_{3}$ (independent of $u(\cdot)$ and of $\lambda$ ) such that

$$
\begin{gathered}
\|u\|_{\infty}<R_{2}, \\
\left\|u^{\prime}\right\|_{2}<\lambda R_{3} .
\end{gathered}
$$

Step 2. Lower bounds for $m=\min \{|u(t)|: t \in[0, T]\}$ follow from (G1), (G2) and Step 1.

As we assume that (G1) and the strong force condition (G2) hold, the lower bounds for $m$ follow from Lemma 6 of Habets and Sanchez [5] by noticing the estimate (2.11) and the estimate (2.12) (with the factor $\lambda$ ). Namely, there is some $R_{4}>0$ (independent of $u$ and of $\lambda$ ) such that

$$
\min \{|u(t)|: t \in[0, T]\}>R_{4} .
$$

Step 3. Upper bounds for $\left\|u^{\prime}\right\|_{\infty}$ follow from Steps 1 and 2. 
By (2.11) and (2.13), we have

$$
R_{4}<|u(t)|<R_{2} \quad \forall t \in[0, T] .
$$

Thus there is some $c_{4}>0$ such that, for all $t \in[0, T]$,

$$
|G(u(t))| \leq c_{4}
$$

and

$$
\left|(\nabla F(u(t)))^{\prime}\right|=\left|\frac{\partial^{2} F}{\partial u^{2}}(u(t)) u^{\prime}(t)\right| \leq c_{4}\left|u^{\prime}(t)\right| .
$$

Now we use (2.5) to get

$$
\begin{aligned}
\left\|u^{\prime \prime}\right\|_{1} & =\lambda\left|\int_{0}^{T}\left((\nabla F(u))^{\prime}+\nabla G(u)-h(t)\right) d t\right| \\
& \leq \int_{0}^{T}\left(c_{4}\left|u^{\prime}\right|+c_{4}+|h(t)|\right) d t \\
& =c_{4}\left\|u^{\prime}\right\|_{1}+T c_{4}+\|h\|_{1} \\
& <\sqrt{T} R_{3} c_{4}+T c_{4}+\|h\|_{1}=: R_{5} .
\end{aligned}
$$

For each $i=1, \ldots, N$, there exists some $t_{i} \in(0, T)$ such that $u_{i}^{\prime}\left(t_{i}\right)=0$ because $u_{i}(0)=u_{i}(T)$. Thus, for any $t \in[0, T]$

Therefore,

$$
\left|u_{i}^{\prime}(t)\right|=\left|\int_{t_{i}}^{t} u_{i}^{\prime \prime}(s) d s\right| \leq\left\|u_{i}^{\prime \prime}\right\|_{1}<R_{5}
$$

$$
\left\|u^{\prime}\right\|_{\infty}=\max _{t \in[0, T]}\left(\sum_{i=1}^{N}\left|u_{i}^{\prime}(t)\right|^{2}\right)^{\frac{1}{2}}<\sqrt{N} R_{5}=: R_{6} .
$$

Step 4. The existence result follows from (G5) and Steps 1-3.

For the completion of the proof, we need only take the domain in Mawhin's result, noting the estimates $(2.11),(2.13)$ and $(2.14)$, as

$$
\Omega=\left\{(x, y) \in \mathbb{R}^{N} \times \mathbb{R}^{N}: R_{4}<|x|<R_{2},|y|<R_{6}\right\},
$$

because the degree condition (G5) is assumed.

Remarks. 1. When $N=1$, a different estimating routine is given in [4], [9], [11] because the solutions $u(\cdot)$ do not cross the singularity 0 . In this case, the strong force condition (G2) is unnecessary.

2. Theorem 1 says that if $G(u)$ satisfies some strong force condition at the singularity 0, the existence of periodic solutions to Eq. (2.1) can be obtained provided that the potential $G(u)$ is smaller than the first eigenvalue of the corresponding Dirichlet problem at infinity. Meanwhile, no restriction on the damping term $F(u)$ is imposed. Such a result may compare with the result of Majer [7] for undamped differential systems with attractive singular forces:

$$
u^{\prime \prime}+\nabla G(u)=h(t) \text {. }
$$

He used Ljusternik-Schnirelmann theory to obtain the existence of periodic solutions to Eq. (2.15) when $G(u)$ satisfies some strong force condition at the singularity 0 and $G(u)$ is smaller than the first eigenvalue of the Dirichlet problem at infinity. 


\section{Concluding Remarks}

In this section we will give a discussion on our assumptions (G1)-(G5) and derive some results from Theorem 1 .

1. The condition (G1) implies, by a conventional trick, that, for any $m>0$ one has

$$
G(u)>c_{5}-m \log |u|, \quad 0<|u| \ll 1 .
$$

Thus, $\lim _{u \rightarrow 0} G(u)=+\infty$, which means that $G(u)$ is of repulsive type.

2. Condition (G3) is concerned with the growth of $G(u)$ at infinity, because (G1) implies that (2.3) holds when $|u|$ is small.

3. The potentials $G(u)=|u|^{-\gamma}$ satisfy (G1) and (G3) for all $\gamma>0$, while Habets-Sanchez's strong force condition (G2) holds only when $\gamma \geq 2$.

4. (G4) can be implied by each of the following conditions (independent of $F$ ):

(G4) ${ }^{\prime}$ There is some constant $R_{7}>0$ such that, for any $C^{1} T$-periodic function $u$ satisfying

$$
\frac{1}{T} \int_{0}^{T} \nabla G(u(t)) d t=\bar{h}
$$

one has

$$
|u(\tau)|<R_{7}
$$

for some $\tau \in[0, T]$.

$(\mathrm{G} 4)^{\prime \prime}$ There is some constant $R_{7}>0$ such that

$$
|\nabla G(u)|<|\bar{h}|, \quad \forall|u| \geq R_{7} .
$$

It is obvious that $(\mathrm{G} 4)^{\prime \prime}$ implies $(\mathrm{G} 4)^{\prime}$. Assume now that $(\mathrm{G} 4)^{\prime}$ holds. Then, for any solution $u$ to $(2.5)-(2.6)$, the integration of (2.5) over $[0, T]$ yields (3.1). So the condition (G4) holds by (3.2).

Under the condition (G4)", we have the following result as a corollary of Theorem 1.

Theorem 2. Assume that $G$ and $h$ satisfy (G1), (G2) and (G4)". Then (2.1)-(2.2) has at least one solution.

Proof. (G1) and (G4)" imply that (G3) holds for any $0<c_{2} \ll 1$. On the other hand, the degree condition (G5) can be obtained by introducing the following homotopy

$$
\mu \nabla G(u)+(1-\mu) \nabla G_{0}(u), \quad \mu \in[0,1],
$$

where $G_{0}(u)=|u|^{-\gamma}, \gamma>0$. As $\bar{h} \neq 0$ by (3.3), we can compute that

$$
\operatorname{deg}\left(\nabla G_{0}, \Omega_{r, R}, \bar{h}\right) \neq 0
$$

Remarks. 1. Theorem 2 shows that the assumption (A-1), i.e., the dissipative condition (1.3) or (1.4), in [5, Theorem 1] for the repulsive case is unnecessary and $h \in L^{1}$ is also allowed.

2. When $F(u) \equiv 0$, Theorem 2 is just Theorem 3 in [5]. 


\section{REFERENCES}

[1] A. Ambrosetti, Critical Points and Nonlinear Variational Problems, Mem. Soc. Math. France, 49 (1992). MR 93g:58024

[2] V. Coti Zelati, Dynamical systems with effective-like potentials, Nonlinear Anal. 12 (1988), 209-222. MR 89c:58034

[3] W. B. Gordon, Conservative dynamical systems involving strong forces, Trans. Amer. Math. Soc. 204 (1975), 113-135. MR 51:14152

[4] P. Habets and L. Sanchez, Periodic solutions of some Liénard equations with singularities, Proc. Amer. Math. Soc. 109 (1990), 1035-1044. MR 90k:34049

[5] P. Habets and L. Sanchez, Periodic solutions of dissipative dynamical systems with singular potentials, Differential Integral Equations 3 (1990), 1139-1149. MR 92a:34040

[6] A. C. Lazer and S. Solimini, On periodic solutions of nonlinear differential equations with singularities, Proc. Amer. Math. Soc. 99 (1987), 109-114. MR 87k:34064

[7] P. Majer, Ljusternik-Schnirelmann theory with local Palais-Smale conditions and singular dynamical systems, Ann. Inst. H. Poincaré Anal. Non Linéaire 8 (1991), 459-476. MR 93e: 58024

[8] J. Mawhin, Topological Degree Methods in Nonlinear Boundary Value Problems, CBMSRegional Conf. Math. no. 40, Amer. Math. Soc., Providence, RI, 1979. MR 80c:47055

[9] J. Mawhin, Topological degree and boundary value problems for nonlinear differential equations, in Topological Methods for Ordinary Differential Equations (M. Furi and P. Zecca, Eds.), pp. 74-142, Lecture Notes in Math. vol 1537, Springer-Verlag, New York/Berlin, 1993. MR 94h:47121

[10] S. Solimini, On forced dynamical systems with a singularity of repulsive type, Nonlinear Anal. 14 (1990), 489-500. MR 91d:58041

[11] M. Zhang, Periodic solutions of Liénard equations with singular forces of repulsive type, J. Math. Anal. Appl. 203 (1996), 254-269. MR 97m:34079

Department of Applied Mathematics, Tsinghua University, Beijing 100084, People's Republic of China

E-mail address: mzhang@math.tsinghua.edu.cn 